\title{
EFEITO DA MATURAÇÃO E TEMPERATURA NA GERMINAÇÃO DE SEMENTES DE Phoenix canariensis hort. ex Chabaud - ARECACEAE ${ }^{1}$
}

\author{
Ricardo Soares Pimenta ${ }^{2}$, Petterson Baptista da Luz ${ }^{3}$, Kathia Fernandes Lopes Pivetta ${ }^{2}$, Amanda de \\ Castro $^{2}$ e Patricia Unger César Pizetta ${ }^{2}$
}

\begin{abstract}
RESUMO - Phoenix canariensis hort. ex Chabaud, originária das Ilhas Canárias, é uma palmeira que apresenta grande valor ornamental. A propagação das palmeiras, de modo geral, é considerada lenta, desuniforme e influenciada por vários fatores, como estádio de maturação e temperatura. Devido à sua importância e à falta de informações na literatura sobre a propagação da espécie, este trabalho teve como objetivo estudar o efeito do estádio de maturação e da temperatura na germinação de sementes de $P$. canariensis. Realizou-se um experimento cujo delineamento experimental foi inteiramente casualizado em esquema fatorial 2 x 5 ( 2 para maturação e 5 para temperatura), com quatro repetições de 25 sementes. Frutos de colorações alaranjada (intermediário) e marrom (maduro) foram despolpados e os diásporos, colocados em caixas plásticas (tipo gerbox) contendo vermiculita como substrato, nas temperaturas de $25,30,35,20-30$ e $25-35^{\circ} \mathrm{C}$, com fotoperíodo de $16 \mathrm{~h}$ de luz e $8 \mathrm{~h}$ de escuro, utilizando-se câmaras incubadoras tipo BOD com controle de temperatura e fotoperíodo. Pelos resultados, conclui-se que a condição que permitiu maior porcentagem de germinação das sementes de P. canariensis foi a partir de frutos maduros (de coloração marrom), na temperatura alternada de $20-30{ }^{\circ} \mathrm{C}$, atingindo $98 \%$ de germinação.
\end{abstract}

Palavras-chave: Palmeira, Falsa-tamareira e Propagação.

\section{EFFECT OF MATURATION STAGE AND TEMPERATURE ON GERMINATION OF Phoenix canariensis Hort. ex Chabaud (Arecaceae) seeds}

\begin{abstract}
Phoenix canariensis Hort. ex Chabaud is a palm tree of great ornamental value native to the Canary Islands. Its commercial propagation is done by sexual means and there are only few studies on seed germination under the influence of various factors such as maturation stage and temperature. We tried to evaluate the seed germination of $P$. canariensis using fruits at different maturarion stages under different temperatures. The experiment was arranged in a complete randomized design in a $2 x 5$ factorial scheme (two maturation stages and five temperature conditions), with four samples containing 25 seeds each. Brownish and orangish fruits had their pulp removed and their germinative capacity was evaluated by a germination test conducted in $B O D$ incubation chambers under the temperatures of $25^{\circ} \mathrm{C}, 30^{\circ} \mathrm{C}, 35^{\circ} \mathrm{C}, 20-30^{\circ} \mathrm{C}$ and 25 $35^{\circ} \mathrm{C}$, and photoperiod of 16L:8D. Seeds were sown on vermiculite. The results obtained in this experiment led to the conclusion that the alternate temperatures of $20-30^{\circ} \mathrm{C}$ were the best for the germination of $\boldsymbol{P}$. canariensis seeds from mature fruits ( brownish fruits), resulting in $98 \%$ of germination.
\end{abstract}

Keywords: Palm tree, Canary Island date palm and Propagation.

\section{INTRODUÇÃO}

Phoenix canariensis hort. ex Chabaud é uma palmeira dioica, solitária, robusta, muito rústica, originária das Ilhas Canárias. Adapta-se a climas e solos variados, inclusive a climas temperados e aos solos pobres em matéria orgânica; além disso, resiste à seca e aos ventos marinhos (LORENZI e MELLO FILHO, 2001). Apresenta raízes fasciculadas expostas na base, seus frutos são ovoides, amarelo-alaranjados,

\footnotetext{
${ }^{1}$ Recebido em 12.06.2007 e aceito para publicação em 14.10.2009.

${ }^{2}$ Universidade Estadual Paulista Júlio de Mesquita Filho, Faculdade de Ciências Agrárias e Veterinárias de Jaboticabal, Departamento de Produção Vegetal - Jaboticabal, SP - Brasil E-mail: <kathia@fcav.unesp.br>.

${ }^{3}$ Universidade do Estado de Mato Grosso, Campus Universitário de Cáceres, Departamento de Agronomia, Caceres, MT Brasil. E-mail: <petterbaptista@yahoo.com.br>.
} 
produzidos mesmo na ausência da planta masculina. Embora apresente características ornamentais notáveis e seja de introdução antiga no Brasil, não é muito difundida (LORENZI et al., 2004), e um dos motivos pode ser a falta de mudas no mercado. Na literatura há poucas informações sobre propagação dessa espécie.

A propagação comercial das palmeiras é feita quase que exclusivamente por sementes; geralmente a porcentagem de germinação é baixa e ocorre de forma lenta e desuniforme, sendo influenciada por vários fatores, como estádio de maturação ideal para a colheita dos frutos e temperatura durante o processo de germinação (PIVETTA et al., 2007; MEEROW, 1991).

A determinação da época de colheita das sementes de uma espécie pode ser facilitada pela adoção de índices de maturação, ou seja, de parâmetros práticos que permitem inferir o estádio de desenvolvimento do fruto e da semente, entre os quais figuram modificações visíveis no aspecto externo dos frutos e das sementes (RODRIGUES e AGUIAR, 1993).

Com relação as palmeiras, Lorenzi et al. (2004) comentaram que os melhores resultados são obtidos com sementes provenientes de frutos maduros, sendo a germinação de sementes de frutos imaturos muito falha, podendo até não ocorrer, porque o endosperma se encontra ainda aquoso, não solidificado.

Trabalhos realizados com algumas espécies de palmeiras têm mostrado variações na germinação em função do estádio de maturação. Pivetta et al. (2005c), analisando a germinação de sementes de Syagrus schizophylla provenientes de frutos verdes, amarelos ou avermelhados, concluíram que os melhores resultados foram obtidos com frutos amarelos ou avermelhados. Já os melhores resultados obtidos de Livistona chinensis (MACIEL, 1996) foram a partir de frutos colhidos verdes.

De acordo com Bewley e Black (1985), a temperatura influencia tanto a velocidade quanto a porcentagem final de germinação. As sementes são capazes de germinar sob determinada amplitude de temperatura, definida para cada espécie, existindo uma temperatura máxima e uma mínima; na Família Arecaceae, há variações de temperatura desde 24 até $35^{\circ} \mathrm{C}$ (MEEROW, 1991; BROSCHAT, 1994; LORENZI et al., 2004).
Generalizando, Meerow (1991) comentou que temperaturas entre 20 e $40{ }^{\circ} \mathrm{C}$ são aceitáveis para que ocorra a germinação de sementes de palmeiras, porém, melhores resultados são obtidos entre $30-35{ }^{\circ} \mathrm{C}$ da maioria das espécies. Já Lorenzi et al. (2004) relatam que em várias espécies de palmeiras são favoráveis temperaturas entre $24-28{ }^{\circ} \mathrm{C}$ e umidade do ar em torno de $70 \%$.

Mwale et al. (1994) comentaram que a faixa de temperatura apresentada nas espécies de regiões tropicais é de $15 \mathrm{a} 40^{\circ} \mathrm{C}$, como foi verificado por Mello e Barbedo (2007) na germinação de sementes de paubrasil (Caesalpinia echinata), embora $25^{\circ} \mathrm{C}$ tenham sido a temperatura que proporcionou melhores resultados.

Embora a maioria das palmeiras seja de origem tropical e as sementes geralmente germinam, naturalmente em temperaturas mais elevadas, muitos trabalhos testando temperaturas variadas evidenciaram que maior porcentagem de germinação têm sido encontrada em diferentes espécies, como $35^{\circ} \mathrm{C}$ para Acoelorraphe wrightii, Coccothrinax argentata, Sabal etonia, Thrinax morrisii e Thrinax parviflora (CARPENTER, 1988; PIVETTA et al., 2005a), 25 e 30 ${ }^{\circ} \mathrm{C}$ para Phoenix roebelenii (IOSSI et al., 2003), 25 ${ }^{\circ} \mathrm{C}$ para Rhapis excelsa (AGUIAR et al., 2005), $30 \mathrm{e}$ $35^{\circ} \mathrm{C}$ para Syagrus romanzoffiana (PIVETTA et al., 2005b) e temperaturas alternadas de $30-35^{\circ} \mathrm{C}$ para Chrysalidocarpus lutescens (BROSCHAT e DONSELMAN, 1986) e $20-30{ }^{\circ} \mathrm{C}$ e $25^{\circ} \mathrm{C}$ para Euterpe edulis (ANDRADE et al., 1999).

De modo geral, recomenda-se colher das sementes das palmeiras quando os frutos estiverem bem maduros, o que se reconhece pela mudança de coloração e desprendimento do cacho, no entanto é importante associar essas características com a maturidade fisiológica das sementes (PIVETTA et al., 2007).

Chapin (1999), estudando a fenologia de oito espécies de palmeiras, utilizou como critérios de indicação de maturação dos frutos a coloração do epicarpo, a consistência pastosa do mesocarpo e a queda dos frutos ou deiscência.

Aguiar et al. (2005) estudaram a germinação de sementes de Rhapis excelsa, de frutos em três estádios de maturação, amarelos, intermediários e 
castanhos; as sementes foram colocadas para germinar em três temperaturas, constantes de 25 e $35^{\circ} \mathrm{C}$ e alternada de $25-35^{\circ} \mathrm{C}$, com e sem luz, em três substratos, areia, vermiculita e turfa. Os melhores resultados foram os com frutos amarelos, a $25^{\circ} \mathrm{C}$, sem luz e substrato areia.

Devido à importância e a falta de informações na literatura sobre a propagação desta espécie, este trabalho teve como objetivo estudar o efeito do estádio de maturação e da temperatura e na germinação de sementes de $P$. canariensis.

\section{MATERIAL E MÉTODOS.}

Sementes de $P$. canariensis foram obtidas por meio de coleta de cachos de 10 matrizes presentes no paisagismo da cidade de Jaboticabal $\left(21^{\circ} \mathrm{S}, 48^{\circ} \mathrm{W}\right)$, SP.

Os frutos foram separados dos cachos e agrupados em duas categorias, de acordo com sua coloração externa, em alaranjado (intermediário) e marrom (maduro). Em seguida, foram imersos em água por 24 h, para a retirada do epicarpo e mesocarpo e os diásporos (sementes com o endocarpo aderido), assim obtidos, foram secos à sombra.

O delineamento experimental foi inteiramente casualizado em esquema fatorial 2 x 5 ( 2 estádios de maturação caracterizados pela coloração externa dos frutos: alaranjada e marrom e cinco condições de temperatura: constantes de $25,30,35^{\circ} \mathrm{C}$ e alternadas de $20-30^{\circ} \mathrm{C}$ e $25-35^{\circ} \mathrm{C}$ ), com quatro repetições de 25 sementes.

Os diásporos foram colocados em caixas plásticas transparentes com tampa, nas dimensões de 11 x 11 x $3 \mathrm{~cm}$ (tipo gerbox) utilizando vermiculita como substrato. As caixas foram colocadas em germinadores cujas temperaturas foram reguladas de acordo com o tratamento, com fotoperíodo de $8 \mathrm{~h}$ de luz e $16 \mathrm{~h}$ de escuro. O teste foi conduzido durante 40 dias, até a estabilização da germinação, verificada pela emissão do botão germinativo.

Determinou-se a porcentagem de germinação calculada pela fórmula proposta nas Regras para Análise de Sementes (BRASIL, 1992):

$$
\mathrm{G}=\frac{\mathrm{NG} \times 100}{\mathrm{NT}}
$$

em que:

$\mathrm{NG}=$ número de sementes germinadas; e

$\mathrm{NT}=$ número de sementes colocadas para germinar

O Índice de Velocidade de Germinação (IVG) foi calculado utilizando-se a fórmula proposta por Maguire (1962):

$$
\mathrm{IVG}=\Sigma \frac{\mathrm{Ngi}}{\mathrm{Ti}}
$$

em que:

$\mathrm{NGi}=$ número de sementes germinadas no dia i; e

Ti = tempo, em dias, após a semeadura, para a germinação.

Os dados foram submetidos à análise de variância, sendo os de porcentagem de germinação previamente transformados em arco-seno "x/100 e analisados estatisticamente; as médias comparadas pelo teste de Scott-Knott a 5\% de probabilidade.

\section{RESULTADO E DISCUSSÃO}

Tanto para a porcentagem quanto para o Índice de Velocidade de Germinação (IVG) a interação entre temperatura e estádio de maturação foi significativa. As sementes provenientes de frutos alaranjados, nas temperaturas de $30,20-30$ e $25-35^{\circ} \mathrm{C}$, foram as com maior porcentagem de germinação e IVG. Já as dos frutos marrons, maior porcentagem a $20-30{ }^{\circ} \mathrm{Ce} 25-35^{\circ} \mathrm{C}$ e maior IVG a $25,20-30{ }^{\circ} \mathrm{C}$ e $25-35^{\circ} \mathrm{C}$ (Tabela 1 ).

A condição que permitiu maior porcentagem de germinação das sementes de $P$. canariensis foi a partir de frutos de coloração marrom, ou seja, no estádio de maturação mais avançado, na temperatura alternada de 20-30 ${ }^{\circ} \mathrm{C}$, atingindo $98 \%$ de germinação, seguida de 25 $35{ }^{\circ} \mathrm{C}$, com $89 \%$ de germinação.

Estes resultados estão de acordo com os comentários de Pivetta et al. (2007) de que é recomendado colher as sementes de palmeiras quando os frutos estiverem bem maduros, e neste caso, pelos resultados obtidos, sementes de $P$. canariensis atingiram o ponto de maturidade fisiológica quando os frutos encontravam na coloração marrom.

R. Árvore, Viçosa-MG, v.34, n.1, p.31-38, 2010 
Tabela 1 - Quadrados médios e médias obtidas da porcentagem de germinação e Índice de Velocidade de Germinação (IVG) de sementes de Phoenix canariensis submetidas a dois estádios de maturação e cinco diferentes temperaturas. Jaboticabal, SP, 2006.

Table 1 - Means and mean squares of germination percentage and speed of germination index (IVG) of Phoenix canariensis seeds submitted to 2 maturation stages and 5 different temperatures. Jaboticabal, SP, 2006.

\begin{tabular}{|c|c|c|c|}
\hline Fontes de variação & GL & Porcentagem de germinação $^{1}$ & $\mathrm{IVG}^{2}$ \\
\hline Estádio de maturação (E) & 1 & $1109,7094 *$ & $0,4487 *$ \\
\hline Temperatura $(\mathrm{T})$ & 4 & $1291,9102 *$ & $1,4086 *$ \\
\hline $\mathrm{E} \times \mathrm{T}$ & 4 & $283,8206^{*}$ & $0,3207 *$ \\
\hline Repetição & 3 & 91,3760 & 0,0741 \\
\hline Resíduo & 27 & 44,6319 & 0,0667 \\
\hline $\mathrm{CV}(\%)$ & & 15,49 & 29,40 \\
\hline \multicolumn{4}{|l|}{ Médias } \\
\hline Alaranjado $-25^{\circ} \mathrm{C}$ & & $19,00^{1}(10,60)^{2} b$ & $0,3819 \mathrm{~b}$ \\
\hline Alaranjado $-30^{\circ} \mathrm{C}$ & & $52,00(62,10) \mathrm{a}$ & $1,0225 \mathrm{a}$ \\
\hline Alaranjado - 35 & & $15,00(6,70) b$ & $0,2256 \mathrm{~b}$ \\
\hline Alaranjado $-20-30{ }^{\circ} \mathrm{C}$ & & $54,00(65,45)$ a & $1,1704 \mathrm{a}$ \\
\hline Alaranjado $-25-35^{\circ} \mathrm{C}$ & & $55,00(67,10) \mathrm{a}$ & $1,0626 \mathrm{a}$ \\
\hline Marrom $-25^{\circ} \mathrm{C}$ & & $61,00^{1}(76,50)^{2}$ b & $1,1586 \mathrm{~b}$ \\
\hline Marrom $-30{ }^{\circ} \mathrm{C}$ & & $44,00(48,26) \mathrm{c}$ & $0,7041 \mathrm{c}$ \\
\hline Marrom $-35^{\circ} \mathrm{C}$ & & $20,00(11,70) \mathrm{d}$ & $0,2730 \mathrm{~d}$ \\
\hline Marrom - 20-30 ${ }^{\circ} \mathrm{C}$ & & $81,00(97,55)$ a & $1,5014 \mathrm{a}$ \\
\hline Marrom - 25-3 $5^{\circ} \mathrm{C}$ & & $71,00(89,40)$ a & $1,2852 \mathrm{a}$ \\
\hline
\end{tabular}

As médias nas colunas e dentro dos grupos seguidas da mesma letra não diferem entre si, pelo teste de Scott Knott a $5 \%$ de probabilidade.

${ }^{1}$ Dados transformados em arco-seno "x/100.

${ }^{2}$ Dados não transformados.

Também Chapin (1999), estudando a maturação dos frutos de oito espécies de palmeiras, utilizou a coloração do epicarpo como um dos critérios de indicação de fruto maduro.

Aguiar et al. (2005) também estudaram o efeito da temperatura e do estádio de maturação na germinação de sementes de Rhapis excelsa, onde os melhores resultados foram com frutos amarelos (intermediários) a $25^{\circ} \mathrm{C}$.

O efeito da temperatura na germinação de sementes de várias espécies de palmeiras foi estudado por Broschat e Donselman (1986), Carpenter (1988), Andrade et al. (1999), Iossi et al. (2003), Pivetta et al. (2005a) e Pivetta et al. (2005b), porém não fizeram correlações com o estádio de maturação. De forma inversa, sem correlacionar com a temperatura Pivetta et al. (2005c) estudaram o efeito do estádio de maturação.

As diferentes faixas de temperaturas empregadas influenciaram diferentemente nos dois estágios de maturação; porém, as temperaturas alternadas de 20$30{ }^{\circ} \mathrm{C}$ e $25-35{ }^{\circ} \mathrm{C}$ proporcionaram maiores médias de porcentagem de germinação e IVG.

R. Árvore, Viçosa-MG, v.34, n.1, p.31-38, 2010
Nos gráficos das Figuras 1 e 2, observa-se que a germinação de sementes de $P$. canariensis foi bastante desuniforme reforçando os comentários de Meerow (1991).

A germinação das sementes dos frutos alaranjados (Figura 1) iniciou a partir do quinto dia para as temperaturas de $30,20-30{ }^{\circ} \mathrm{C}$ e $25-35^{\circ} \mathrm{C}$ e no sétimo dia para as de 25 e $35^{\circ} \mathrm{C}$. Já nas sementes dos frutos marrons (Figura 2) a partir do nono dia em todas as temperaturas.

O pico de germinação foi variável nas diferentes temperaturas, porém, a grande maioria das sementes, independentemente da temperatura, germinou do quinto ao vigésimo dia, mostrando que, embora a germinação tenha sido desuniforme, foi rápida quando comparada com outras espécies da família Arecacaeae, como Euterpe edulis, cuja germinação finalizou aos 98 dias, ou seja, 14 semanas (ANDRADE et al., 1999).

Andrade et al. (1999) comentaram sobre a importância de padronizar os métodos de análise de sementes para as espécies que não constam nas Regras para Análise de Sementes (BRASIL, 1992) e baseados nos gráficos de germinação ao longo do tempo, indicaram a sétima e $14^{a}$ semanas como contagem inicial e final nos testes de germinação de sementes de Euterpe edulis. 


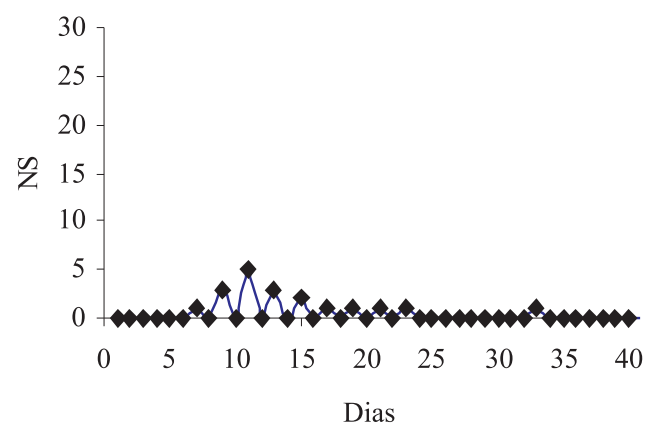

$25^{\circ} \mathrm{C}$

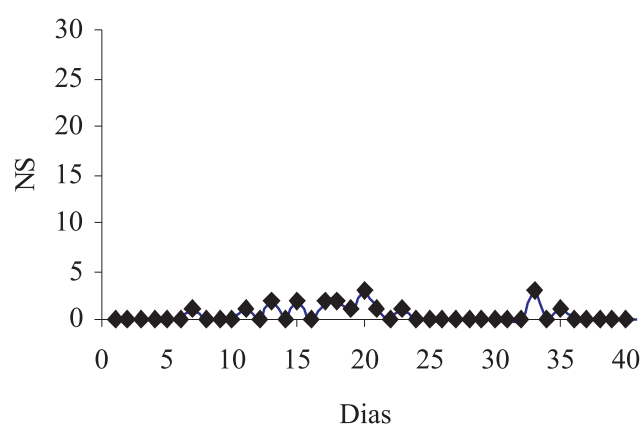

$35^{\circ} \mathrm{C}$

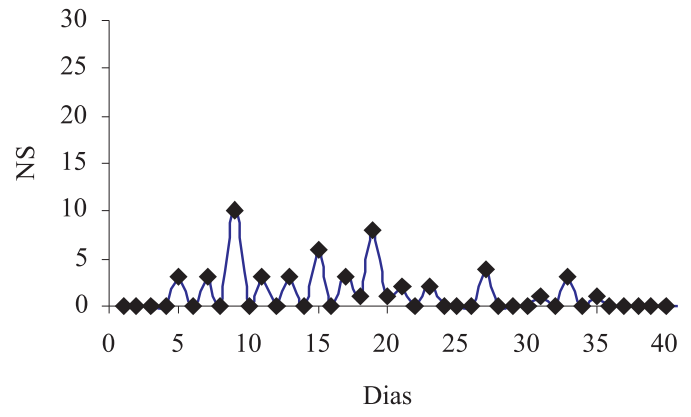

$30{ }^{\circ} \mathrm{C}$

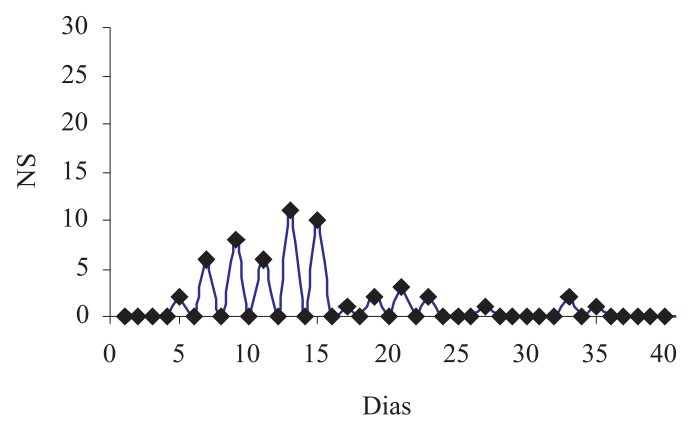

20-30 ${ }^{\circ} \mathrm{C}$

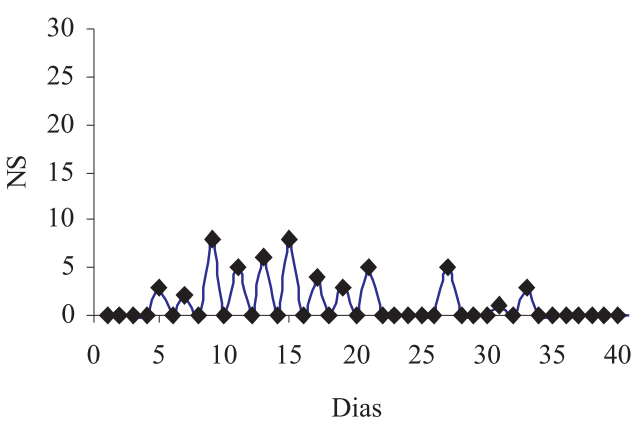

\section{$25-35{ }^{\circ} \mathrm{C}$}

Figura 1 - Distribuição da germinação de sementes (NS - número de sementes germinadas $\operatorname{dia}^{-1}$ ) ao longo de 35 dias, de um lote de 100 sementes de Phoenix canariensisi em diferentes condições de temperatura, a partir de frutos de coloração alaranjada. Jaboticabal, SP, 2006.

Figure 1 - Distribution of seed germination (NS - number of germinated seeds day ${ }^{-1}$ ) during 35 days, from one lot of 100 seeds of Phoenix canariensisi in different conditions of temperature at the orangish stage of maturation. Jaboticabal, SP, 2006. 


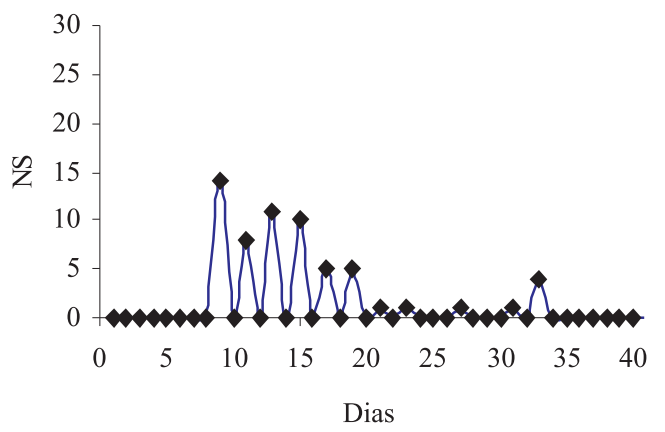

$25^{\circ} \mathrm{C}$

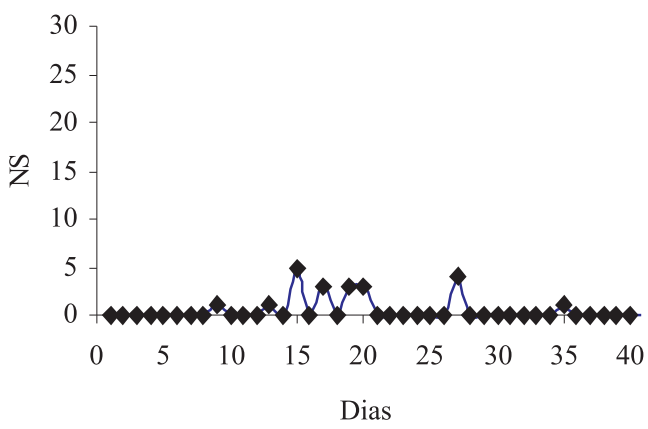

$35^{\circ} \mathrm{C}$

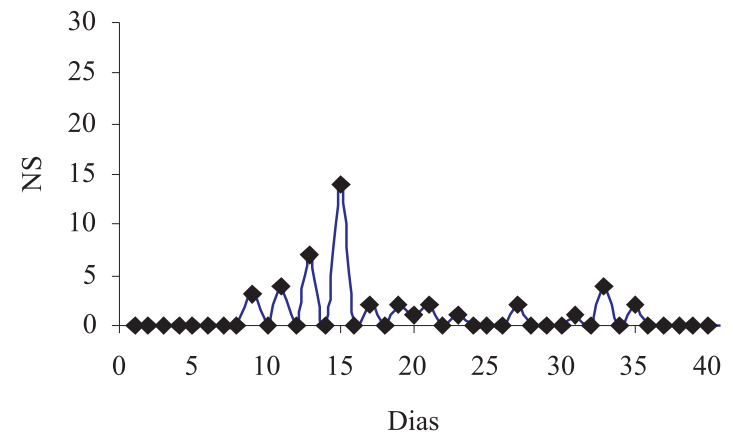

$30{ }^{\circ} \mathrm{C}$

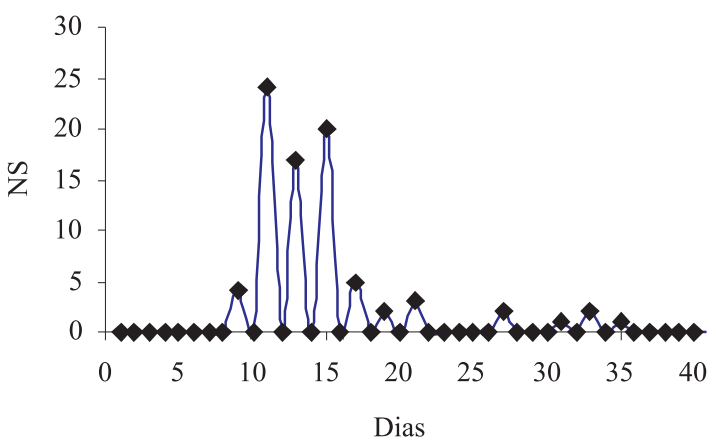

20-30 ${ }^{\circ} \mathrm{C}$

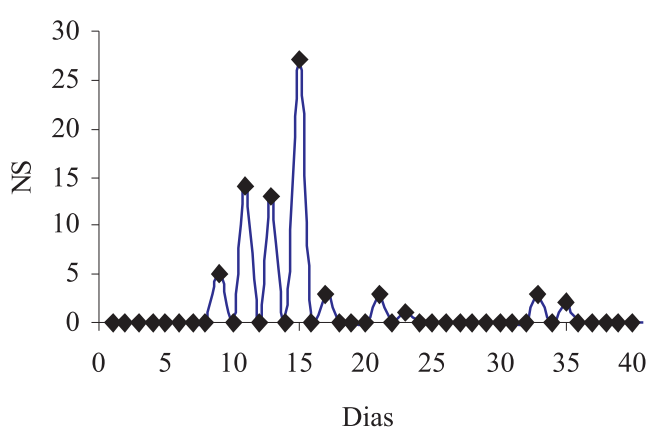

$25-35{ }^{\circ} \mathrm{C}$

Figura 2 - Distribuição da germinação de sementes (NS - número de sementes germinadas dia $^{-1}$ ) ao longo de 35 dias, de um lote de 100 sementes de Phoenix canariensis em diferentes condições de temperatura, a partir de frutos de coloração marrom. Jaboticabal, SP, 2006.

Figure 2-Distribution of seed germination (NS - number of germinated seeds day ${ }^{-1}$ ) during 35 days, from one lot of 100 seeds of Phoenix canariensisi in different conditions of temperature and at the brownish stage of maturation. Jaboticabal, SP, 2006.

R. Árvore, Viçosa-MG, v.34, n.1, p.31-38, 2010 
Dessa forma, considerando o melhor resultado obtido neste estudo (frutos marrons a 20-30 ${ }^{\circ} \mathrm{C}$ ), há indicativo de contagens inicial e final, respectivamente, no nono e $35^{\circ}$ dia.

\section{CONCLUSÃO}

A condição que permitiu maior porcentagem de germinação das sementes de $P$. canariensis foi a partir de frutos de coloração marrom, na temperatura alternada de $20-30{ }^{\circ} \mathrm{C}$, atingindo $98 \%$ de germinação.

\section{AGRADECIMENTOS}

À Fapesp, pelo auxílio-pesquisa.

\section{REFERENCIAS}

AGUIAR, F. F. A. et al. Germinação de sementes de Rhapis excelsa (Thunb.) Henry ex Rehder: efeitos da temperatura, luz e substrato. Hoehnea, v.32, n.1, p.119-126, 2005.

ANDRADE, A. C. S. et al. Reavaliação do efeito do substrato e da temperatura na germinação de sementes de palmiteiro (Euterpe edulis Mart.). Revista Árvore, v.23, n.3, p.279-283, 1999.

BEWLEY, J. D.; BLACK, M. Physiology and biochemistry of seeds. Berlim: SpringerVerlag, 1985. v.1. 540p.

BRASIL. Ministério da Agricultura. Regras para análise de sementes. Brasília: Secretaria Nacional de Defesa da Agropecuária, 1992. 365p.

Broschat, T. K. Palm seed propagation. Acta Horticulturae, n.360, p.141-147, 1994.

BROSCHAT, T. K.; DONSELMAN, H. Factors affecting storage and germination of Chrysalidocarpus lutescens seeds. Journal of American Society of

Horticultural Science, v.111, n.6, p.872$877,1986$.

CARPENTER, W. J. Temperature affects seed germination of four Florida palm species. HortScience, v.23, n.2, p.336-337, 1988.
CHAPIN, M. H. Flowering and fruiting phenology in certain palms. Palms, v.43, n.4, p.161-165, 1999.

IOSSI, E. et al. Efeitos de substratos e temperaturas na germinação de sementes de tamareira-anã (Phenix roebelenii O'Brien).

Revista Brasileira de Sementes, v.25, n.2, p.63-69, 2003.

LOREnZI, H. et al. Palmeiras brasileiras e exóticas e cultivadas. Nova Odessa: Instituto Plantarum, 2004. 416p.

LORENZI, H.; MELLO FILHO, L. E. As plantas tropicais de Roberto Burle Marx; the tropical plants of R. Burle Marx. São Paulo: Instituto Plantarum de Estudos da Flora, 2001. 488p.

MACIEL, N. M. S. Efectos de la madurez y el almacenamiento del fruto, la escarificacion y el remojo de las semillas sobre la emergencia de la palma china de abanico. Agronomia Tropical, v.46, n.2, p.155-170, 1996.

MAGUIRE, J. D. Speed of germination aid in selection and evaluation for seedling emergence and vigor. Crop Science, v.2, n.2, p.176-177, 1962 .

MEerow, A. W. Palm seed germination. Florida: Cooperative Extension Service, 1991. 10p. (Bulletin, 274)

MELLO, J. I. O.; BARBEDO, C. J. Temperatura, luz e substrato para germinação de sementes de paubrasil (Caesalpinia echinata Lam., Leguminosae Caesalpinioideae). Revista Árvore, v.31, n.4, p.645-655, 2007.

MWALE, S. S. et al. Effect of temperature pn the germination of sunflower (Helianthus annuus L.). Seed Science and Technology, v.22, p.565571, 1994.

PIVETTA, K. F. L. et al. Efeito da temperatura e do armazenamento na germinação de sementes de Thrinax parviflora Swartz. (Arecaceae). Científica, v.33, n.2, p.178-184, 2005a.

R. Árvore, Viçosa-MG, v.34, n.1, p.31-38, 2010 
PIMENTA, R.S. et al.

PIVETTA, K. F. L. et al. Effects of maturation and scarification on seed germination of Syagrus schizophylla (Mart.) Glass.

(Arecaceae). Acta Horticulturae, v.683, p.375-378, 2005c.

PIVETTA, K. F. L. et al. Propagação de palmeiras e estrelitzias. In: BARBOSA, J.G.; LOPES, L.C.

Propagação de plantas ornamentais. Viçosa, MG: Universidade Federal de Viçosa, 2007. p.43-70.
PIVETTA, K. F. L. et al. Effects of temperature on seed germination of Queen Palm Syagrus romanzoffiana (Cham.) Glassman. (Arecaceae). Acta Horticulturae, v.683, p.379-381, 2005b.

RODRIGUES, F. C. M.; AGUIAR, I. B. Maturação e dispersão de sementes. In: AGUIAR, I. B.; PIÑA-RODRIGUES, F. C. M.; FIGLIOLIA, M. B. (Eds.). Sementes florestais tropicais. Brasília: Associação Brasileira de Tecnologia de Sementes, 1993. p.215-274. 